\title{
Hemodialysis-Catheter Related Septic Pulmonary Emboli in the Absence of Endocarditis or Right Atrial Thrombus
}

\author{
Udip Dahal $^{\mathrm{a}}$, Bandana Pathak ${ }^{\mathrm{a}}$, Vijaya Raj Bhatt ${ }^{\mathrm{a}}$, Srujitha Murukutla, ${ }^{\mathrm{a},}$, Shiksha Kediaa, \\ Shradha Pant ${ }^{b}$, Suzanne El-Sayegh ${ }^{c}$, Neville Mobaraki ${ }^{\mathrm{d}}$
}

\begin{abstract}
Catheter-related bloodstream infection, although a major cause of morbidity and mortality in hemodialysis population, is rarely associated with septic pulmonary emboli (SPE). SPE is generally associated with endocarditis or right atrial thrombus. We describe a case of a young man on hemodialysis with fever, who was subsequently diagnosed to have SPE in the absence of endocarditis or right atrial thrombus.
\end{abstract}

Keywords: Septic pulmonary emboli; Endocarditis; Catheter related infection

\section{Introduction}

Different types of intravascular catheters are used worldwide, with $>150$ million users just in the US alone, for a variety of medical reasons including hemodialysis (HD) [1]. With an increasing pandemic of end-stage kidney disease and an increased use of hemodialysis catheters, there can be an expected rise in infectious complications including catheter-related bloodstream infections (CRBSI) among end-stage kidney disease (ESKD) patient population [2]. Although CRBSI is common, catheter-related septic pulmonary emboli (SPE) is unusual especially without endocarditis

\footnotetext{
Manuscript accepted for publication May 10, 2012

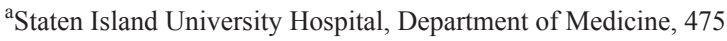
Seaview Avenue, Staten Island New York, USA

${ }^{\mathrm{b}}$ University of Science and Technology, Department of Internal Medicine, Chittagong 4202, Bangladesh

${ }^{\mathrm{c} S t a t e n}$ Island University Hospital, Department of Medicine, Division of Nephrology 475 Seaview Avenue, Staten Island, New York, USA ${ }^{\mathrm{d}}$ Department Of Medicine, Staten Island University Hospital, Staten Island, New York, USA

${ }^{e}$ Corresponding author: Srujitha Murukutla, Staten Island University Hospital, Department of Medicine, 475 Seaview Avenue, Staten Island, New York, 10305, USA. Email: srujitha.murukutla@gmail.com
}

doi: http://dx.doi.org/10.4021/jmc684w or infected right atrial thrombus [3-11].

\section{Case Report}

A 44-year-old man presented to the emergency department with two-day history of sudden onset of fever associated with chills, rigors, generalized headache and an episode of vomiting. He denied any neck pain, cough, shortness of breath, chest pain, abdominal pain, diarrhea, any urinary symptoms, seizure, or altered sensorium. He had a past history of polyarticular gout, hypertension, end stage kidney disease secondary to idiopathic focal segmental glomerulosclerosis, anemia of chronic disease and appendectomy. He had undergone placement of tunneled Ash catheter in the right internal jugular vein two months ago for hemodialysis and was awaiting a renal transplantation. His medications included amlodipine, clonidine, labetalol, calcium acetate, allopurinol and prednisone $20 \mathrm{mg}$ daily, which he has been taking for more than 2 years. He lived in New York with his wife. He denied recent travel, as well as recent contact with a sick person or a pet. He drank alcohol occasionally but did not smoke or use illicit drugs. He had a history of allergy to morphine sulphate.

Physical examination revealed a temperature of 103.8 ${ }^{\circ} \mathrm{F}$, blood pressure of $132 / 76 \mathrm{~mm} \mathrm{Hg}$, pulse of 139 /min, respiratory rate of $16 / \mathrm{min}$ and oxygen saturation of 96 percent on ambient air. The lungs were clear on auscultation; cardiac examination revealed no murmurs. Neurological exam was unremarkable. Ash catheter exit site or tunnel infection was absent. There were no Osler's nodes, Jane way lesions, conjuctival petechiae, splinter hemorrhage or Roth spots. Rest of the physical examination was normal.

Laboratory tests revealed: white cell count of $6900 / \mu \mathrm{L}$ with $65 \%$ neutrophils and $26 \%$ bands, hemoglobin of $9 \mathrm{gm} /$ $\mathrm{dl}$ (which was at his baseline) with mean corpuscular volume of $80 \mathrm{fl}$, platelet count of $116000 / \mu \mathrm{L}$, blood urea nitrogen of $83 \mathrm{mg} / \mathrm{dl}$, creatinine of $13.4 \mathrm{mg} / \mathrm{dl}$, normal electrolytes and liver function tests. The urine analysis was negative for pyuria or hematuria. Chest x-ray of the patient showed a questionable left upper lobe infiltration (Fig. 1). Blood cultures were drawn. Computed tomography (CT) of head was nor- 


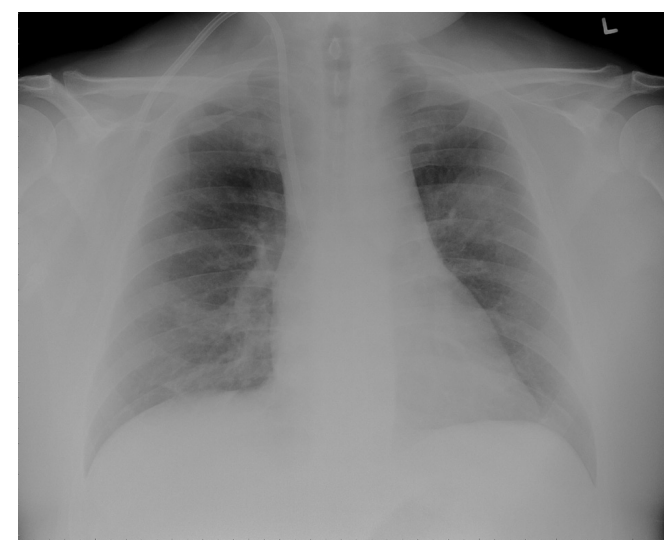

Figure 1. Chest $X$-ray showing left upper lobe mass like infiltrate and a tunneled ash catheter in the right internal jugular vein.

mal. Cerebrospinal fluid (CSF) analysis showed: clear fluid with white cell count of $13 / \mu \mathrm{L}$ with $90 \%$ neutrophils, red cell count of $34500 / \mu \mathrm{L}$, total protein of $64 \mathrm{mg} / \mathrm{dl}$, glucose of $72 \mathrm{mg} / \mathrm{dl}$ (peripheral blood glucose $149 \mathrm{mg} / \mathrm{dl}$ ) and negative gram stain and India ink.

Patient was started on intravenous (IV) cefepime, linezolid and moxifloxacin for possible health-care associated pneumonia. CT chest revealed multiple areas of peripherally located soft tissue densities within the lungs, the largest one being $4 \mathrm{~cm} \times 2.9 \mathrm{~cm}$ in size. The findings were consistent with septic embolization (Fig. 2, 3). The blood culture grew methicillin resistant staphylococcus aureus (MRSA). CSF culture was negative. The patient was started on IV vancomycin $750 \mathrm{mg}$ with hemodialysis after a one time loading dose of $2 \mathrm{gm}$ and all other antibiotics were discontinued. Given the possibility of CRBSI leading to SPE, Ash catheter was removed. Culture of the catheter tip grew MRSA confirming the diagnosis. Transthoracic echocardiogram

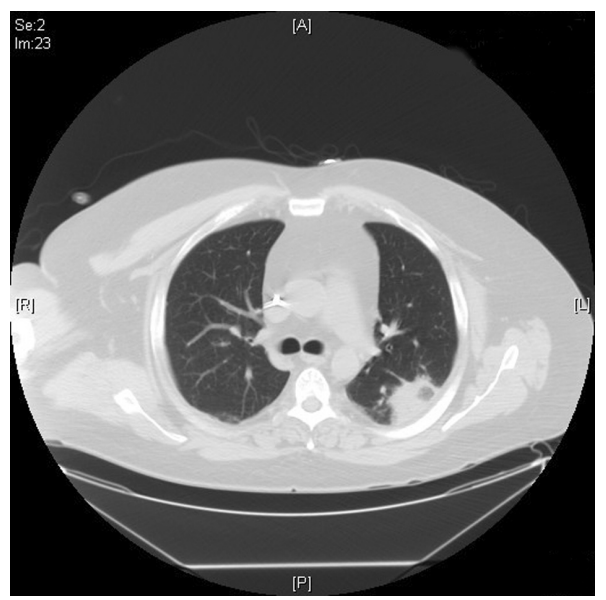

Figure 2. CT chest showing $4 \mathrm{~cm} \times 2.9 \mathrm{~cm}$ density in the superior segment of the left lower lung field.

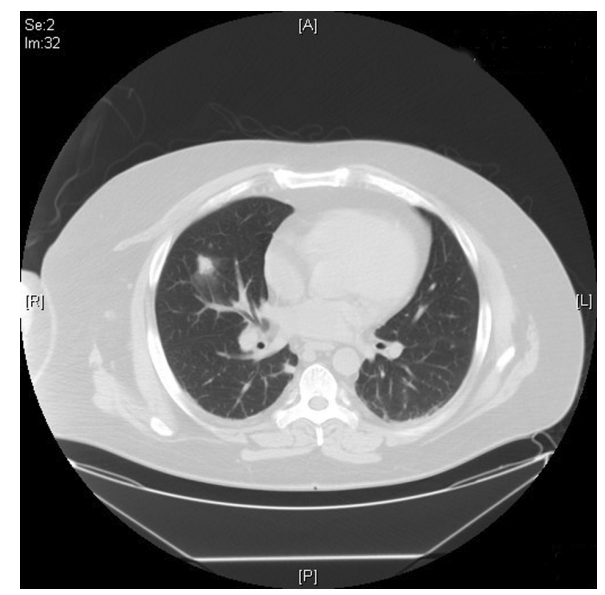

Figure 3. CT chest showing $1.7 \mathrm{~cm} \times 1.3 \mathrm{~cm}$ right mid-lung field soft tissue density.

(TTE) as well as transesophageal echocardiogram (TEE) did not reveal any vegetation or intracardiac thrombus (Fig. 4). The patient's clinical status improved. Repeat blood cultures done 3 days after the initiation of antibiotics and 1 day after catheter removal were negative. The patient subsequently underwent placement of Ash catheter in right internal jugular vein and was discharged home on day 11 to complete 6 weeks of IV vancomycin as outpatient. Weekly blood cultures sent for 4 weeks after the completion of the antibiotic course were negative and the patient continues to do well 2 months after the discharge.

\section{Discussion}

SPE is unusual, with a large autopsy study of 396,982 postmortem examination showing an incidence of $2.2 \%$ of the total PE cases $(n=11,367)$. The majority of 247 cases of SPE were associated with pneumonia, sepsis and infectious endo-

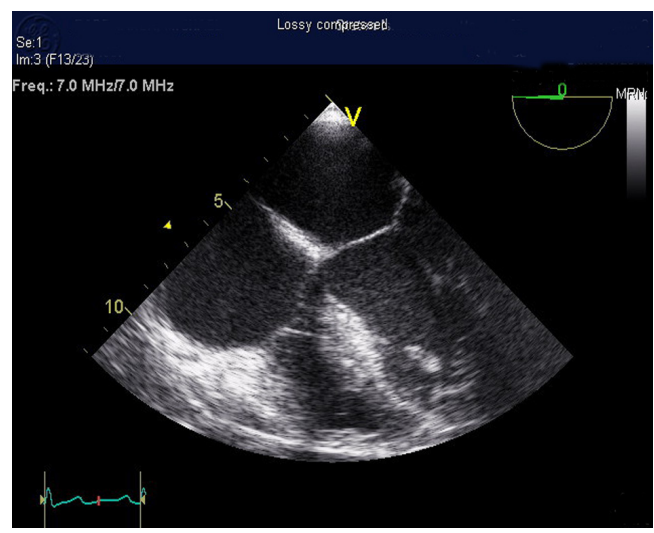

Figure 4. Transesophageal echocardiogram showing no evidence of valvular vegetation or cardiac thrombus. 
carditis with only one case associated with infected central venous catheter, thus showing the rarity of the catheter-related SPE. In this study, fungal SPE was twice more common then bacterial SPE and more likely to be associated with hematological or solid malignancy compared to bacterial SPE [12]. In another study, tricuspid endocarditis was the most common cause and the drug abusers were the most common patients [13].

CRBSI occurs at the frequency of 0.9 to 2.0 episodes/ patient-year among HD population.Ten persent of whom would be hospitalized with sepsis or metastatic infection (such as septic thrombosis, endocarditis and septic arthritis) [2]. Although CRBSI is very common, catheter-related SPE is unusual, more so in the absence of endocarditis or infected right atrial thrombus [3-11].

The pathogenesis of SPE is related to the formation of infected catheter-induced thrombosis at the proximal end of the catheter, often with extension of the thrombus into the right atrium, which is frequently visualized by an echocardiogram. Alternatively, there could be associated tricuspid endocarditis. Dislodgement of the thrombus or vegetation to the pulmonary artery or its branches can lead to an infarction or a metastatic abscess [3, 14-16]. Our patient developed multiple SPE in the absence of overt clinical or radiographic signs of catheter-associated thrombosis, catheter infection or malfunction or endocarditis. Therefore, it is possible that the patient might have developed small thrombosis around the catheter site without extension into right atrium or the patient might have dislodged the entire cardiac thrombus without any residual thrombus.

SPE typically presents with fever, pleuritic pain, cough and hemoptysis. There is often a history of predisposing factor, e.g. drug abuse or extra pulmonary infection $[13,14]$ or a catheter, as in our patient. The clinical features and chest radiography could be nonspecific, as in our patient, thus delaying the diagnosis. High index of suspicion is important since SPE due to central venous catheters is probably under diagnosed [15] and early identification may improve its prognosis. Equally important is the identification of septic focus since the resolution of the problem will depend on the elimination of the source. When chest radiograph findings are nonspecific or equivocal, the use of chest CT can play an important role in detecting the presence of SPE. Only $32 \%$ of lesions visible on chest CTs were detected by chest radiographs in a study [17]. Chest CT might show patchy infiltrate, poorly defined nodule or wedge-shaped densities, which are typically multiple, sub pleural and peripheral, and located at the end of a blood vessel [13, 14, 17], and sometimes associated with cavitations and air bronchogram within the nodule, ground-glass halo around the nodule, pleural effusion or empyema $[13,14]$. These features, along with the clinical scenario, help to differentiate SPE from other causes of diffuse pulmonary nodules [18]. Echocardiogram is valuable in detecting the presence of right-atrial thrombus or en- docarditis; TEE is superior to TTE [14]. Given the rarity of catheter-related SPE, we believe that the following criteria should be fulfilled before the catheter can be definitely implicated as the source of septic emboli: SPE occurs in the presence of CRBSI, and there are no other possible sources of septic emboli such as soft-tissue infection or drug abuse.

Once CRBSI is suspected, prompt empiric therapy with antibiotic covering methicillin resistant staphylococcus aureus (preferably vancomycin) and gram-negative bacilli (e.g. third generation cephalosporin, carbapenem, or beta-lactam/ beta-lactamase inhibitor combination) should be started [1, 2]. The infected catheter should always be removed for patients with infection due to staphylococcus aureus, pseudomonas species, or Candida species and a temporary (nontunneled catheter) should be inserted into another anatomical site. The catheter should also be removed in the presence of severe sepsis, suppurative thrombophlebitis, or metastatic infection e.g. endocarditis, or osteomyelitis or in the absence of response to antibiotic therapy within 72 hours [1]. Hence, in patients with SPE, the catheter should be removed. Our patient had infection with MRSA as well as SPE, hence the catheter was removed. Long-term HD catheter can be reinserted once blood cultures are negative as in our patient. The total duration of antibiotic in the presence of complicated or metastatic infection should be at least 6 weeks [1]. The need of surgical interventions for complications of SPE such as lung abscesses, empyema and bronchopleural fistula, although once common [13], is declining with earlier diagnosis and prompt use of antibiotics [14].

\section{Conclusions}

1) The diagnosis of SPE might be overlooked because of the absence of respiratory symptoms and signs or the presence of nonspecific clinical and radiological findings. Hence, a high index of suspicion should be maintained in patients with HD catheter. 2) A diagnosis of CRBSI can be made by peripheral blood culture in conjunction with catheter tip or catheter blood culture. A chest CT, in addition to echocardiogram, can provide important additional diagnostic clues for SPE. 3). Empiric therapy of CRBSI should include vancomycin and gram-negative coverage with subsequent deescalation based on cultures. SPE requires six-week course of antibiotics.

\section{Grant}

None.

\section{Competing Interests}

The authors declare that they have no competing interests. 


\section{References}

1. Mermel LA, Allon M, Bouza E, Craven DE, Flynn P, O'Grady NP, Raad, II, et al. Clinical practice guidelines for the diagnosis and management of intravascular catheter-related infection: 2009 Update by the Infectious Diseases Society of America. Clin Infect Dis. 2009;49(1):1-45.

2. Allon M. Dialysis catheter-related bacteremia: treatment and prophylaxis. Am J Kidney Dis. 2004;44(5):779791.

3. Abid Q, Price D, Stewart MJ, Kendall S. Septic pulmonary emboli caused by a hemodialysis catheter. Asian Cardiovasc Thorac Ann. 2002;10(3):251-253.

4. Cook RJ, Ashton RW, Aughenbaugh GL, Ryu JH. Septic pulmonary embolism: presenting features and clinical course of 14 patients. Chest. 2005;128(1):162-166.

5. Faisal W, Burnton G, Imlay-Gillespie L, Robilliard J. Cerebral abscesses and septic pulmonary emboli due to Serratia marcescens infection arising from a Portacath. J Microbiol Immunol Infect. 2010;43(6):538-541.

6. Sozener ZC, Kayal A, Atasoy C, Kilickap M, Numanoglu N, Savas I. Septic pulmonary embolism: three case reports. Monaldi Arch Chest Dis. 2008;69(2):75-77.

7. Wong KS, Lin TY, Huang YC, Hsia SH, Yang PH, Chu SM. Clinical and radiographic spectrum of septic pulmonary embolism. Arch Dis Child. 2002;87(4):312315.

8. Cervia JS, Caputo TA, Davis SD, Murray HW. Septic pulmonary embolism complicating a central venous catheter. Chest. 1990;98(6):1526.

9. Goodwin NJ, Castronuovo JJ, Friedman EA. Recurrent septic pulmonary embolism complicating maintenance hemodialysis. Ann Intern Med. 1969;71(1):29-38.

10. Leiby JM, Purcell H, DeMaria JJ, Kraut E, Sagone AL, Metz EN. Pulmonary embolism as a result of Hickman catheter-related thrombosis. Am J Med. 1989;86(2):228231.

11. Shiba E, Kambayashi J, Sakon M, Yoshida T, Taniguchi K, Kawasaki T, Arisawa J, et al. Septic pulmonary emboli after prolonged use of central venous catheter for parenteral nutrition. Case report. Eur J Surg. 1992;158(1):59-61.

12. Sakuma M, Sugimura K, Nakamura M, Takahashi T, Kitamukai O, Yazu T, Yamada N, et al. Unusual pulmonary embolism: septic pulmonary embolism and amniotic fluid embolism. Circ J. 2007;71(5):772-775.

13. MacMillan JC, Milstein SH, Samson PC. Clinical spectrum of septic pulmonary embolism and infarction. $\mathrm{J}$ Thorac Cardiovasc Surg. 1978;75(5):670-679.

14. Jorens PG, Van Marck E, Snoeckx A, Parizel PM. Nonthrombotic pulmonary embolism. Eur Respir J. 2009;34(2):452-474.

15. Rello J. Pulmonary embolism due to an indwelling central venous catheter. Chest. 1991;100(5):1477-1478.

16. Sajjad A, Carey PA, Arnold IR, Smith SR. Infected right atrial thrombus presenting as pulmonary embolism complicating central venous cannulation. BMJ. 1995;311(7015):1288.

17. Iwasaki Y, Nagata K, Nakanishi M, Natuhara A, Harada $\mathrm{H}$, Kubota Y, Yokomura I, et al. Spiral CT findings in septic pulmonary emboli. Eur J Radiol. 2001;37(3):190194.

18. Boitsios G, Bankier AA, Eisenberg RL. Diffuse pulmonary nodules. AJR Am J Roentgenol. 2010;194(5):W354366. 